Approximate solution of a Thomas-Fermi model equation for bulk self-gravitating stellar objects in two dimensions

This content has been downloaded from IOPscience. Please scroll down to see the full text.

2017 Eur. J. Phys. 38015406

(http://iopscience.iop.org/0143-0807/38/1/015406)

View the table of contents for this issue, or go to the journal homepage for more

Download details:

IP Address: 129.16.69.49

This content was downloaded on $02 / 12 / 2016$ at $17: 23$

Please note that terms and conditions apply.

You may also be interested in:

Introduction to direct variational and moment methods and an application to the Child-Langmuir law

D Anderson and M Desaix

Scattering on two Aharonov-Bohm vortices

E Bogomolny

Thomas-Fermi model for a bulk self-gravitating stellar object in two dimensions

Sanchari De and Somenath Chakrabarty

Numerical methods for finding stationary gravitational solutions

Óscar J C Dias, Jorge E Santos and Benson Way

Normalized functionals of first passage Brownian motion and a curious connection with the maximal relative height of fluctuating interfaces

Michael J Kearney and Richard J Martin

Asymptotics semiclassically concentrated on curves for the nonlocal

Fisher-Kolmogorov-Petrovskii-Piskunov equation

E A Levchenko, A V Shapovalov and A Yu Trifonov

Polarised black holes in AdS

Miguel S Costa, Lauren Greenspan, Miguel Oliveira et al.

Parity horizons in shape dynamics

Gabriel Herczeg

A note on the Fourier transform

M L Glasser 


\title{
Approximate solution of a Thomas-Fermi model equation for bulk self-gravitating stellar objects in two dimensions
}

\author{
D Anderson ${ }^{1,3}$ and M Desaix ${ }^{2}$ \\ ${ }^{1}$ Department of Earth and Space Sciences, Chalmers University of Technology \\ University of Technology, SE-412 96 Göteborg, Sweden \\ ${ }^{2}$ Faculty of Textiles, Engineering and Business, University College of Borås, SE-501 \\ 90 Borås, Sweden \\ E-mail: elfda@chalmers.se
}

Received 12 September 2016, revised 12 October 2016

Accepted for publication 31 October 2016

Published 30 November 2016

\begin{abstract}
Direct variational methods are used to find simple approximate solutions of the Thomas-Fermi equations describing the properties of self-gravitating radially symmetric stellar objects both in the non-relativistic and ultra-relativistic cases. The approximate solutions are compared and shown to be in good agreement with exact and numerically obtained solutions.
\end{abstract}

Keywords: direct variational methods, Rayleigh-Ritz optimization procedure, Thomas-Fermi model equation

(Some figures may appear in colour only in the online journal)

\section{Introduction}

Recently an introductory presentation was given of self-gravitating stellar objects using the Thomas-Fermi model equation for the Fermi energy density in two-dimensional radially symmetric geometry, see [1]. The properties of both non-relativistic and ultra-relativistic cases were studied and analysed. In the non-relativistic case, the characteristic equation is a linear equation, admitting explicit analytical solutions. However, in the ultra-relativistic case, a non-linear equation determines the properties of the stellar object. This equation has no analytical solution and had to be solved numerically. Such situations are met in many physical applications: the linear limit of a problem allows an explicit analytical solution whereas for increasing amplitudes, velocities, densities etc, the characteristic equations become nonlinear

3 Author to whom any correspondence should be addressed. 
and exact analytical solutions are rare. A classical example is the harmonic oscillator for which the small amplitude equation reads

$$
\frac{\mathrm{d}^{2} \theta}{\mathrm{d} t^{2}}+\omega_{0}^{2} \theta=0
$$

where $\theta$ is the swinging angle, $t$ is time and $\omega_{0}$ is the characteristic (low amplitude) oscillation frequency. The solution is well know: $\theta(t)=A \cos \left(\omega_{0} t\right)$ where $A$ denotes the oscillation amplitude. However, for large swinging angles, equation (1) has to be improved to read

$$
\frac{\mathrm{d}^{2} \theta}{\mathrm{d} t^{2}}+\omega_{0}^{2} \sin \theta=0 .
$$

This is a much more complicated (nonlinear) equation for which no simple analytical solution exists and much effort has over the years been devoted to analysing the properties of the resulting nonlinear oscillations, see e.g. [2]. An important new feature associated with the nonlinear case is the fact that, contrary to the linear case, the oscillation frequency now depends on the amplitude.

A powerful and versatile tool for analysis in situations where analytical solutions cannot be found is direct variational methods and in particular the Rayleigh-Ritz optimization procedure, see e.g. [3, 4]. For many problems this makes it possible to find explicit, albeit approximate, analytical solutions which give a clear picture of the properties of the solutions. The accuracy of the obtained approximate solutions can not be determined a priori and the studied equation has also to be solved numerically to assess the quality of the obtained solution. In order to demonstrate the usefulness and the capability of the Rayleigh-Ritz optimization procedure in a manner accessible for under-graduate and graduate students, we will, in the subsequent sections, analyse the Thomas-Fermi equations introduced and studied in [1].

\section{The linear and non-linear Thomas-Fermi equations}

This study will concentrate on the mathematical properties of the model equations analysed in [1] which describes a hypothetical white dwarf star in a two-dimensional radially symmetric geometry. It is shown in [1] that the (normalized) Fermi energy density, here denoted $y(x)$, in the non-relativistic case varies according to the following linear equation

$$
\frac{\mathrm{d}^{2} y}{\mathrm{~d} x^{2}}+\frac{1}{x} \frac{\mathrm{d} y}{\mathrm{~d} x}+y=0,
$$

where $x$ denotes normalized radius. The solution starts at $x=0$ (the centre of the white dwarf) with a finite value of the Fermi energy density and must have vanishing derivative at this point i.e. $\mathrm{d} y(0) / \mathrm{d} x=0$. It then decays to zero at the boundary at some (unknown) radius $x=x_{s}$. The solution of this problem is well known: $y(x)=A J_{0}(x)$ where $A$ denotes the initial value of the Fermi energy density and $J_{0}$ is the lowest order Bessel function. The amplitude, $A$, can be chosen arbitrarily, but the radius of the dwarf, $x_{s}$, is determined by the first zero of the Bessel function i.e. $x_{s} \approx 2.405$. This solution can then be used to determine the properties of the white dwarf e.g. mass-radius relation and average density see [1].

However, in the ultra-relativistic case the corresponding equation for the Fermi energy is a non-linear equation viz. 


$$
\frac{\mathrm{d}^{2} y}{\mathrm{~d} x^{2}}+\frac{1}{x} \frac{\mathrm{d} y}{\mathrm{~d} x}+y^{2}=0
$$

subject to the same boundary conditions as in the non-relativistic case. This equation does not allow an analytical solution and resort is then taken to numerical methods for analysing the properties of the solution see [1].

\section{The Rayleigh-Ritz optimization procedure}

As a first step towards an application of direct variational methods, the problem characterized by equations (1) and (2) must be reformulated as the Euler-Lagrange equations corresponding to a variational problem

$$
\delta \int_{a}^{b} L\left(y, \frac{\mathrm{d} y}{\mathrm{~d} x}\right) \mathrm{d} x=\int_{a}^{b} \frac{\delta L}{\delta y} \delta y \mathrm{~d} x=0
$$

where $L$ is the Lagrangian, $a$ and $b$ are the limits of integration and $\frac{\delta L}{\delta y}$ is the variational derivative which determines the Euler-Lagrange equations according to

$$
\frac{\delta L}{\delta y} \equiv \frac{\partial L}{\partial y}-\frac{\mathrm{d}}{\mathrm{d} x}\left(\frac{\partial L}{\partial\left(\frac{\mathrm{d} y}{\mathrm{~d} x}\right)}\right)=0 .
$$

The so called inverse variational problem i.e. the problem of finding the Lagrangian for which the concomitant Euler-Lagrange equation is equivalent to the considered equation may be more or less difficult, depending on the equation to be studied. However, it is easily shown a posteriori that the Lagrangians corresponding to equations (3) and (4) are respectively

$$
L=\frac{1}{2} x\left(\frac{\mathrm{d} y}{\mathrm{~d} x}\right)^{2}-\frac{1}{2} x y^{2}
$$

and

$$
L=\frac{1}{2} x\left(\frac{\mathrm{d} y}{\mathrm{~d} x}\right)^{2}-\frac{1}{3} x y^{3} .
$$

In the variational principle given by equation (5), the allowed $y(x)$ belong to the set of all sufficiently smooth functions which satisfy the boundary conditions. However, in the Rayleigh-Ritz optimization procedure, they are restrained to a subset of this set by being chosen to have a specific functional dependence on $x$ i.e. $y(x) \rightarrow y_{T}\left(x ; \alpha_{1}, \alpha_{2}, \ldots, \alpha_{n}\right)$ where flexibility is incorporated by allowing dependence on a number of parameters, $\alpha_{k} ; k=1,2, \ldots, n$ which are to be determined by the variational condition. When the trial function $y_{T}\left(x ; \alpha_{1}, \alpha_{2}, \ldots, \alpha_{n}\right)$ is inserted into the Lagrangian, the variational integral becomes a function of the parameters $\alpha_{k}$ only and the optimization condition given by equation (5) reduces to the conditions

$$
\frac{\partial\langle L\rangle}{\partial \alpha_{k}}=0, \quad k=1,2, \ldots, n,
$$

where

$$
\langle L\rangle=\int_{a}^{b} L\left(y_{T}, \frac{\mathrm{d} y_{T}}{\mathrm{~d} x}\right) \mathrm{d} x .
$$


Direct variational methods provide a good complement to numerical methods in situations where the variational optimization in terms of the Euler-Lagrange equations gives rise to difficult differential equations for which simple analytical solutions cannot be found. The choice of suitable trial functions is a crucial qualitative step in the analysis and must be based on the expected general properties of the solution. Although the optimization procedure determines the best choice of trial function, within the chosen form of function, it is not possible to predict, a priori, the quality of the approximation found. This can only be assessed by comparison with the numerical solution. Nevertheless, with a reasonable choice of trial function and the flexibility offered by varying parameters, the subsequent optimization usually provides good and accurate approximations. Many excellent presentations of variational calculus in general and direct variational methods in particular have been given over many years. For the present pedagogical purpose it is referred to the two easily accessible presentations given in $[3,4]$.

\section{Applications to the Thomas-Fermi equations}

Although the non-relativistic case can be solved analytically in terms of the Bessel function $J_{0}(x)$, it is instructive to compare with the result of two simple applications using the direct variational approach. The choice of trial function is made based on the intuitive picture of the solution and is generally a compromise between simplicity and flexibility. As emphasized before, the solution starts at $x=0$ with vanishing derivative and decays to zero at the (unknown) boundary of the white dwarf. In the case of one-dimensional plane geometry, equation (3), reduces to the simple oscillator equation

$$
\frac{\mathrm{d}^{2} y}{\mathrm{~d} x^{2}}+y=0
$$

with the well known solution $y=A \cos x$, which directly determines the boundary width of the dwarf in the plane geometry to be $x_{s}=\pi / 2$. This result makes it tempting to use as trial function, in the cylindrical case, the 'stretched' cos-function $y_{T}(x)=A \cos \left(\frac{\pi}{2} \frac{x}{x_{s}}\right)$ where $x_{s}$ is the new radius (to be determined) in the radially symmetric case. Using this trial function in the Lagrangian given by equation (7) one obtains

$$
\langle L\rangle=\frac{A^{2}}{8}\left(\frac{\pi^{2}}{4}+1\right)-\frac{A^{2} x_{s}^{2}}{2 \pi^{2}}\left(\frac{\pi^{2}}{4}-1\right) .
$$

Variations with respect to $A$ yields

$$
x_{s}=\frac{\pi}{2} \sqrt{\frac{\pi^{2}+4}{\pi^{2}-4}} \approx 2.415 .
$$

Another, more flexible, but still simple trial function satisfying the given boundary conditions is clearly $y_{T}=A\left(1-\frac{x^{2}}{x_{s}^{2}}\right)^{\alpha}$, where $\alpha$ and $A$ are the parameters to be varied. Using this trial function we obtain

$$
\langle L\rangle=\frac{\alpha A^{2}}{2(2 \alpha-1)}-\frac{x_{s}^{2} A^{2}}{4(2 \alpha+1)} .
$$




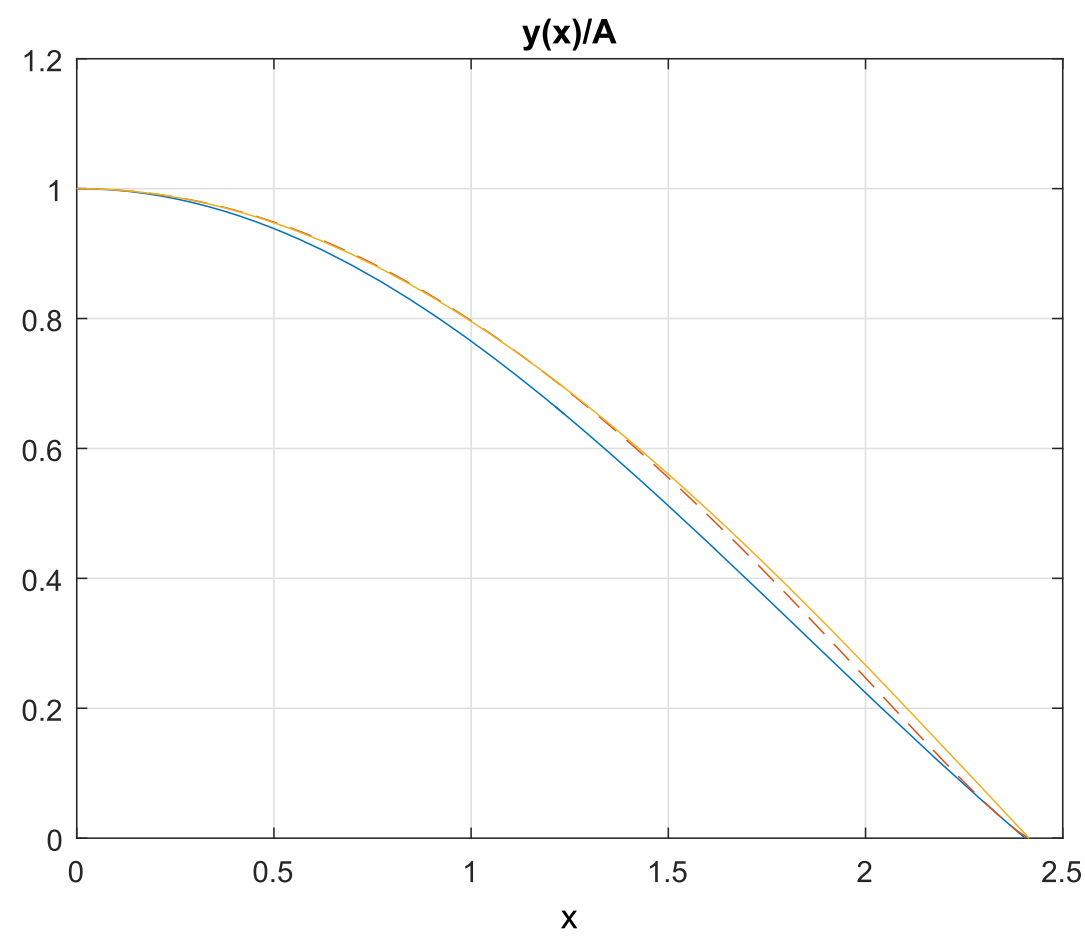

Figure 1. Comparison between the exact solution $y(x) / A=J_{0}(x)$ (lower fully drawn curve), the approximate solution $y(x) / A=\cos \left(\frac{\pi}{2} \frac{x}{x_{s}}\right)$ with the parameter $x_{s}$ is given by equation (13) (fully drawn upper curve) and the approximate solution $y(x) / A=\left(1-x^{2} / x_{s}^{2}\right)^{\alpha}$ (dashed curve) where the parameters $\alpha$ and $x_{s}$ are given by equation (16).

The optimization conditions become

$$
\begin{aligned}
& \frac{\partial\langle L\rangle}{\partial A}=0 \Rightarrow \frac{\alpha}{2 \alpha-1}-\frac{x_{s}^{2}}{2(2 \alpha+1)}=0, \\
& \frac{\partial\langle L\rangle}{\partial \alpha}=0 \Rightarrow-\frac{1}{(2 \alpha-1)^{2}}+\frac{x_{s}^{2}}{(2 \alpha+1)^{2}}=0
\end{aligned}
$$

which can easily be solved to yield

$$
\alpha=(1+\sqrt{2}) / 2 \approx 1.207 ; x_{s}=1+\sqrt{2} \approx 2.414 .
$$

It should be emphasized that the amplitude, $A$, i.e. the central Fermi energy density, can be chosen arbitrarily and does not affect the properties of the solutions (i.e. the parameters $\alpha$ and $x_{s}$ ) - a typical linear feature. The approximate solutions are compared with the exact Bessel solution in figure 1 and show good agreement. In particular, note that the error in the radius of the white dwarf is only $0.4 \%$, the first zero of $J_{0}(x)$ being $x_{s} \approx 2.405$.

Consider now the ultra-relativistic case. The plane geometry version of equation (4) reads

$$
\frac{\mathrm{d}^{2} y}{\mathrm{~d} x^{2}}+y^{2}=0
$$




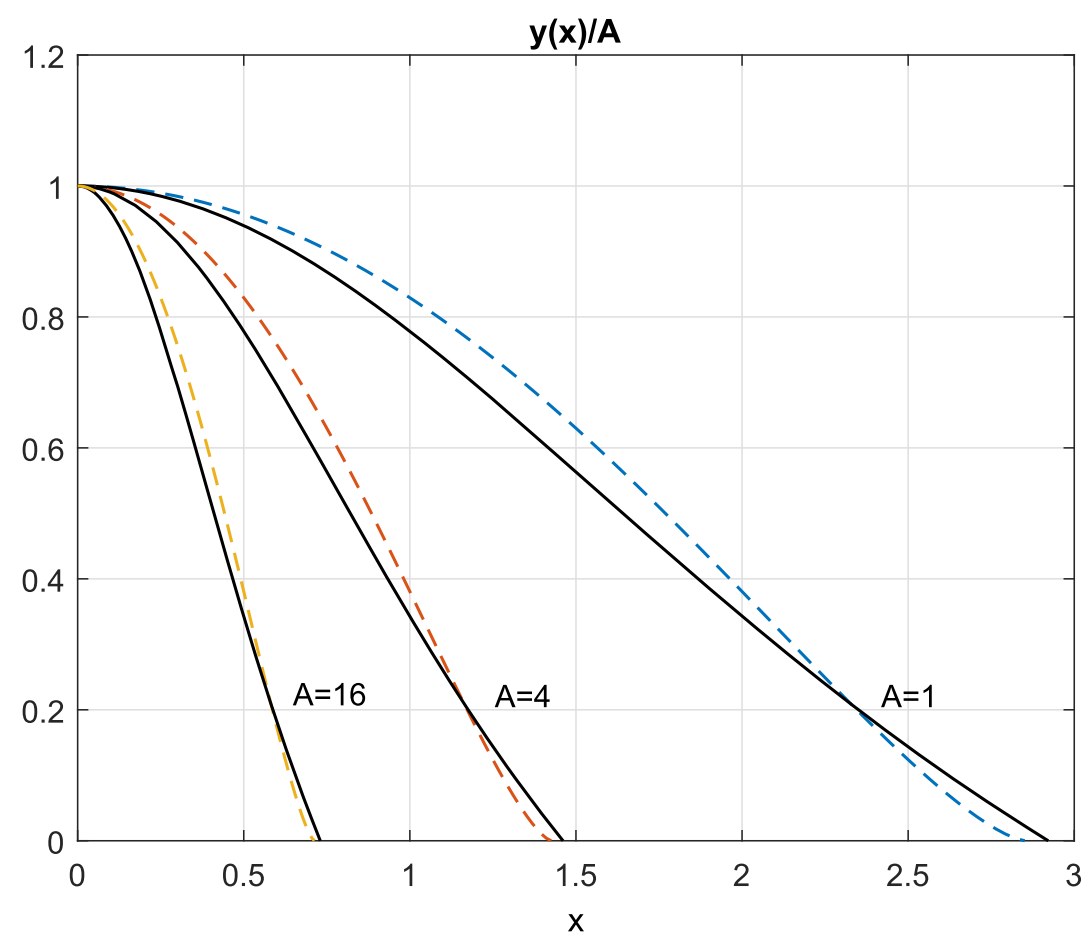

Figure 2. Comparison between the numerically obtained solution $y(x) / A$ (fully drawn) and the approximate solution $y(x) / A=\left(1-x^{2} / x_{s}^{2}\right)^{\alpha}$ (dashed) where the parameters $\alpha$ and $x_{s}$ are given by equation (20).

a nonlinear equation which does have an exact (although complicated) analytical solution in terms of a Weierstrass elliptic function. Unfortunately this solution is not explicit enough to inspire a suitable simple trial function for the radial problem. Instead we use the previous, general and more flexible, trial function $y_{T}=A\left(1-x^{2} / x_{s}^{2}\right)^{\alpha}$ in equation (8). The corresponding integrated Lagrangian is

$$
\langle L\rangle=\frac{\alpha A^{2}}{2(2 \alpha-1)}-\frac{x_{s}^{2} A^{3}}{6(3 \alpha+1)} .
$$

The optimization conditions become

$$
\begin{aligned}
& \frac{\partial\langle L\rangle}{\partial A}=0 \Rightarrow \frac{\alpha}{2 \alpha-1}-\frac{x_{s}^{2} A}{2(3 \alpha+1)}=0, \\
& \frac{\partial\langle L\rangle}{\partial \alpha}=0 \Rightarrow-\frac{1}{(2 \alpha-1)^{2}}+\frac{x_{s}^{2} A}{(3 \alpha+1)^{2}}=0
\end{aligned}
$$

which are easily solved to yield

$$
\alpha=\frac{5+\sqrt{41}}{8} \approx 1.425 ; \quad x_{s} \sqrt{A}=\frac{5+\sqrt{41}}{4} \approx 2.851 .
$$

The amplitude, $A$, can again be chosen arbitrarily and although it does not affect the form of the approximate solution, it determines the radius of the white dwarf. Such a dependence on amplitude is a typical nonlinear feature. The approximate solution is compared with the 
numerically obtained solution in figure 2, again showing good agreement. The obtained approximate value for the product $x_{s} \sqrt{A}$, viz. $x_{s} \sqrt{A} \approx 2.851$ is close to the numerically found value $x_{s} \sqrt{A} \approx 2.921$.

The behaviour of the solution in the ultra-relativistic case is qualitatively different from that in the non-relativistic case. The radius of the white dwarf depends on the central density and decreases as the central energy density increases.

The total Fermi energy (per unit length), $E_{\mathrm{F}}$, is determined by

$$
E_{\mathrm{F}}=2 \pi \int_{0}^{x_{s}} x y(x) \mathrm{d} x
$$

In the non-relativistic case this yields (using for $x_{s}$ the value corresponding to the first zero of $\left.J_{0}(x)\right)$

$$
E_{\mathrm{F}}=2 \pi A \int_{0}^{x_{s}} x J_{0}(x) \mathrm{d} x=2 \pi A x_{s} J_{1}\left(x_{s}\right) \approx 1.25 \cdot 2 \pi A
$$

whereas for the approximate solution we obtain

$$
\begin{aligned}
E_{\mathrm{F}} & =2 \pi A \int_{0}^{x_{s}} x \cos \left(\frac{\pi}{2} \frac{x}{x_{s}}\right) \mathrm{d} x=2 \pi A \frac{2 x_{s}^{2}}{\pi^{2}}(\pi-2) \\
& =2 \pi A\left(\frac{\pi}{2}-1\right) \frac{\pi^{2}+4}{\pi^{2}-4} \approx 1.35 \cdot 2 \pi A \\
E_{\mathrm{F}} & =2 \pi A \int_{0}^{x_{s}} x\left(1-\frac{x^{2}}{x_{s}^{2}}\right)^{\alpha} \mathrm{d} x=2 \pi A \frac{x_{s}^{2}}{2(\alpha+1)} \\
& =2 \pi A \frac{(5+3 \sqrt{2})}{7} \approx 1.32 \cdot 2 \pi A
\end{aligned}
$$

in good agreement with the exact result, the errors being $8 \%$ and $6 \%$ respectively. Note that the total Fermi energy increases linearly with central density $A$.

However, in the ultra-relativistic case, the situation is completely different. The total energy now becomes

$$
E_{\mathrm{F}}=2 \pi A \frac{x_{s}^{2}}{2(\alpha+1)}=\frac{\pi}{4}(7+\sqrt{41}) \approx 1.68 \cdot 2 \pi
$$

i.e. a constant value, independent of the central Fermi energy density. This result is corroborated by the numerical calculations giving $E_{\mathrm{F}} \approx 1.59 \cdot 2 \pi$, implying an error of $6 \%$.

\section{Conclusion}

Direct variational methods play an important role in physics and engineering. An illustrative application has been given to a Thomas-Fermi equation for white dwarfs in cylindrical geometry, a problem recently analysed analytically and numerically in [1]. The aim of the present analysis is to illustrate the power and usefulness of direct variational methods by an application that is easily understandable for under-graduate as well as graduate students. The analysis also emphasizes some important and commonly met differences between the properties of linear and non-linear physical systems. 


\section{References}

[1] De S and Chakrabarty S 2015 Thomas-Fermi model for a bulk self-gravitating stellar object in two dimensions Eur. J. Phys. 36055006

[2] Parwani R R 2004 An approximate expression for the large angle period of a simple pendulum Eur. J. Phys. 25 37-9

[3] Wan F Y M 1995 Introduction to the Calculus of Variations and its Applications (New York: Chapman and Hall)

[4] Komzsik L 2009 Applied Calculus of Variations for Engineers (Boca Raton, FL: CRC Press/Taylor and Francis Group) 